\title{
PRODUÇÃO CIENTÍFICA DOS PROGRAMAS DE PÓS GRADUAÇÃO: AVẢLIAÇÃO DA EFICIÊNCIA DA ÁREA ENGENHARIAS III
}

\author{
M.E.S.S.Vasconcelos ${ }^{1}$; H.R.M.Hora ${ }^{2 *}$; M.Erthal Júnior ${ }^{1,2}$ \\ 1 UCAM-Campos, 28030-335, Campos dos Goytacazes-RJ, Brasil \\ 2 Instituto Federal de Educação, Ciência e Tecnologia Fluminense, 28300-000, Itaperuna - RJ, Brasil \\ *dahora@gmail.com
}

\section{RESUMO}

A avaliação dos cursos de pós-graduação se revela como uma das áreas de maior importância para a aplicação da análise envoltória de dados (DEA). No entanto, para empregar essa metodologia se faz necessário selecionar o conjunto de variáveis: os inputs e outputs que servirão de base para aplicação do método. No Brasil, a entidade responsável por avaliar os cursos de pós-graduação, é a CAPES (Coordenação de Aperfeiçoamento de Pessoal de Nível Superior), cuja avaliação é baseada na atribuição de notas que podem variar de 1 a 5 (no caso em que há apenas o mestrado) ou 7 (quando a Instituição de Educação Superior (IES) apresenta o programa de Doutorado). Diante da real necessidade de verificar a eficiência dos programas de pós-graduação das Engenharias III, o presente artigo sugere o uso da técnica DEA tendo como base os critérios definidos pela CAPES. Encontram-se situações de programas com alto conceito atribuído pela CAPES figuram com baixa eficiência.

\section{SCIENTIFIC PRODUCTION OF GRADUATE PROGRAMS: EFFICIENCY EVALUATION OF ENGINEERING III AREA}

\begin{abstract}
The evaluation of graduate courses is revealed as one of the most important areas for the application of data envelopment analysis (DEA). However, to use this method it is necessary to select the set of variables: the inputs and outputs as a basis for application of the method. In Brazil, the entity responsible for evaluating postgraduate courses is the CAPES (Higher Education Personnel Improvement Coordination), whose
\end{abstract}

assessment is based on grading that can vary from 1 to 5 (in the case where there is only the master) or 7 (when IES presents the doctoral program). Given the real need to check the efficiency of the engineering III postgraduate programs. This article suggests the use of DEA based on the criteria defined by CAPES. They identify situations programs with high concept given by CAPES appearing with low efficiency.

KEYWORDS: Education, Production engineering, Efficiency. 


\section{INTRODUÇÃO}

Até o final do século XVIII, as elites do Brasil buscavam se formar no ensino superior em instituições europeias por serem mais conceituadas mundialmente, porém a partir do início do século XIX a nação brasileira começava a desenvolver a educação de forma mais qualitativa nas universidades consideradas fruto da reunião de alguns institutos isolados no país ou de faculdades específicas de algumas regiões.

Segundo Stallivieri (2009), a educação superior no Brasil desde então vive um processo de expansão e a partir da década de 90 no século XX vem se tornando uma peça chave e de extrema relevância no âmbito do cenário político e social do país.

Ao mesmo tempo em que o governo brasileiro demonstra disposição em efetuar mudanças profundas na política para o ensino em seus diversos níveis, também se faz necessário um aprimoramento dos métodos de diagnóstico e avaliação de desempenho (LINS; ALMEIDA; BARTHOLO JÚNIOR, 2004).

De acordo com Almeida Filho e Ramos (2005), avaliar o desempenho educacional é uma atividade complexa devido a subjetividade que existe na mensuração de fatores como: produção, serviços e produtos.

No Brasil a avaliação dos programas de pós-graduação é feita desde 1976 pela Coordenação de Aperfeiçoamento de Pessoal de Nível Superior - CAPES (ALMEIDA FILHO; RAMOS, 2005), com o objetivo de selecionar os cursos de pós-graduação com vistas à alocação de recursos necessários à consolidação da pós-graduação no Brasil, mediante a concessão de bolsas de estudo e outros tipos de auxílios destinados a estimular a formação de recursos humanos qualificados para a docência no ensino superior, o desenvolvimento da pesquisa científica e o atendimento da demanda de setores não universitários por profissionais com elevada qualificação científica (BELLONI, 2000).

Portanto, entendendo que a eficiência constitui parte fundamental da avaliação da qualidade dos programas de pós-graduação, o presente trabalho objetiva avaliar os indicadores de produção científica dos programas de pós-graduação stricto sensu do Brasil, especificamente a área Engenharias III, composta pelos cursos: Engenharia Aeronáutica, Mecânica, Naval, Oceânica, Petróleo e Produção, tendo como parâmetro os critérios de avaliação estabelecidos pela CAPES (2013).

A utilização do método sugerido permite uma análise quantitativa de cada unidade de decisão (DMU's) obtendo dessa forma um indicador de eficiência com relação aos cursos supracitados.

\section{REVISÃO BIBLIOGRÁFICA}

\subsection{Análise Envoltória de Dados}

A Análise Envoltória de Dados define o posicionamento competitivo relativo de um conjunto de organizações ou atividades contrapondo as suas eficiências ou ineficiências produtivas técnicas, de escalas alocativas (FERREIRA; GOMES, 2012).

A abordagem por Análise Envoltória de Dados (do inglês Data Envelopment Analysis DEA) é uma ferramenta matemática para a medida de eficiência de unidades produtivas (DMU's) (MELLO et al., 2005). A eficiência relativa de uma DMU pode ser definida segundo Angulo-Meza et al. (2003), como a razão da soma ponderada de seus produtos no caso chamados de outputs pela soma ponderada dos inputs (recursos necessários). 
Segundo Ferreira e Gomes (2012), DEA se torna um modelo promissor para avaliação no âmbito competitivo das organizações. Porém avaliar desempenho de uma organização pode não ser uma tarefa fácil de ser realizada, quando se tem vários insumos e produtos.

Lins, Almeida e Bartholo Junior (2005) mostram que o método DEA possui dois modelos clássicos: o modelo CRS ou CCR que foi desenvolvido por Charnes, Cooper e Rhodes (1978) e considera rendimentos de escala constantes ou ainda o modelo VRS ou BBC desenvolvido por Banker, Charnes e Cooper (1984) e que considera rendimentos variáveis de escala.

Vale notar que os o modelo de retorno constante de escala (CRS) é chamado também de CCR por ser esta as iniciais dos autores proponentes. $\mathrm{O}$ mesmo ocorre com o modelo de retorno variável de escala (VRS) sobre BCC. A formulação do modelo linearizado CRS é apresentado por Angulo-Meza et al. (2003), conforme Equação 1.

Equação 1: Modelo CCR.

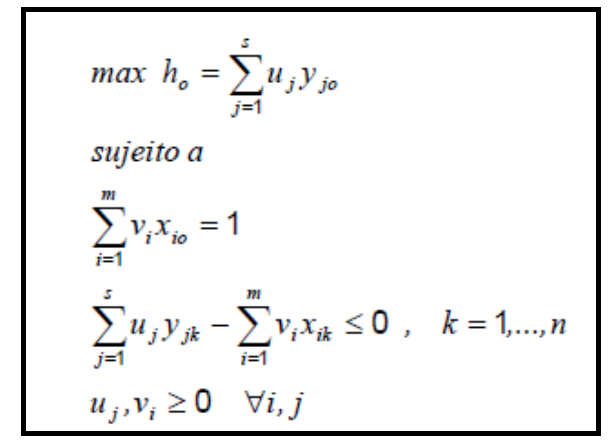

Fonte: Angulo-Meza et al. (2003).

Para esse modelo com n DMU's, m inputs e s outputs, ho é a eficiência da DMU o em análise; xik representa o input i da DMU k, yjk representa o output j da DMU k; vi é o peso atribuído ao input i, uj é o peso atribuído ao output j. Esse modelo deve ser resolvido para cada DMU.

Os resultados do Modelo CCR caracterizam como eficiência produtiva no consumo de recursos (orientada para a redução do consumo de recursos) e eficiência produtiva na produção de resultados (orientada para a maximização da produção de resultados) (CHARNES; COOPER; RHODES, 1978).

Segundo a métrica do DEA, as DMUs $100 \%$ eficientes $(E j=1)$ transformam-se em parâmetros para o modelo, ou seja, constituem a fronteira de eficiência e todos os demais são avaliados em função deles (BARBOSA et al., 2007), e podem ser considerados benchmarking a serem alcançados.

Ao contrário do tratamento analítico da imprecisão da estatística descritiva, onde outliers, também chamados de pontos fora da curva, são higienizados ou corrigidos em uma fase preliminar à aplicação de uma determinada técnica, na Análise Envoltória dos Dados, os pontos fora da curva são, como já dito, a prova da possibilidade de combinar recursos (inputs) para produção de resultados (outputs) em prol de uma melhor produtividade, que deve ser perseguida pelos concorrentes.

Uma fragilidade clássica da DEA é a sua baixa capacidade de ordenar as DMUs, já que quanto maior o número de variáveis em relação ao número de DMUs, menor será a capacidade de ordenação pelas eficiências, já que há a tendência de muitas DMUs ficarem na fronteira (máxima 
eficiência). Um dos métodos usados para contornar este problema é restringir o número de variáveis usadas no modelo. A grande maioria dos trabalhos publicados traz a abordagem da seleção de variáveis segundo a opinião de especialistas ou até mesmo da disponibilidade de dados (SENRA et al., 2007).

\subsection{Avaliação de eficiência do Sistema Nacional de Pós-Graduação (SNPG)}

A história da avaliação das instituições de ensino superior no Brasil não é recente e desenvolveu-se, por muitos anos, por iniciativa quase exclusiva de agências governamentais, de um lado, através de diagnósticos globais do sistema de ensino superior, com o objetivo de formular políticas, e, de outro lado, através de avaliações parciais que se restringem a cursos e programas com o objetivo de subsidiar autorizações, credenciamentos e distribuição de recursos (BELLONI, 2000).

A CAPES é reconhecida como órgão responsável pela elaboração do Plano Nacional de PósGraduação Stricto Sensu, em 1981, pelo Decreto nº 86.791. É também reconhecida como Agência Executiva do Ministério da Educação e Cultura junto ao sistema nacional de Ciência e Tecnologia, cabendo-lhe elaborar, avaliar, acompanhar e coordenar as atividades relativas ao ensino superior (CAPES, 2013).

Ao considerar relevante se ter um sistema de avaliação dos programas de pós-graduação no Brasil, Miranda e Almeida (2003) ressalta a busca pela elevação dos padrões de qualidade desse nível de ensino.

O processo de avaliação da CAPES, inclui critérios qualitativos e quantitativos, contudo ambos são convertidos em considerações qualitativas e, ao final da avaliação, com base nas apreciações realizadas, estabelece-se um conceito numérico dimensionando a qualidade dos programas avaliados (MOREIRA et al., 2011).

Miranda e Almeida (2003) apontam que são atribuídos conceitos entre 1 e 7 aos programas de pós-graduação e aqueles que obtiverem avaliação igual ou superior a 3 tem seus diplomas validados e reconhecidos nacionalmente. Os conceitos atribuídos à programas de mestrado reconhecidos figuram entre 3 e 5 , e os conceitos de programas de doutorado figuram entre 4 e 7 . Os conceitos 1 e 2 não são recomendados pela CAPES, e programas de doutorado que possuem avaliação 3 foram rebaixados na última avaliação e podem ser considerados sob risco de ter seu curso descredenciado na próxima avaliação (que ocorre em 2017).

Dentre os parâmetros utilizados nessa avaliação há critérios relativos à eficiência dos programas, como o tempo de titulação dos mestrandos e doutorandos e a produção bibliográfica dos programas (MOREIRA et al., 2011).

Segundo Belloni (2000) as medidas de eficiência, estão atreladas aos resultados alcançados assim como os recursos utilizados. De acordo com Ferreira e Gomes (2012), podemos definir eficácia como sendo o atendimento de um objetivo e eficiência como uma comparação do que foi produzido - por unidade de inputs como que poderia ter sido produzido.

Lapa e Neiva (1996 apud BALLONI, 2000) descrevem que a eficácia está associada à consecução dos objetivos educacionais propriamente ditos, a efetividade refere-se à consecução de objetivos sociais mais amplos.

A análise envoltória de dados - DEA - se baseia na observação e comparação de dados das organizações ou atividades do conjunto analisado para definir os valores relativos que podem ser produzidos, destacando tanto as eficiências relativas de cada organização ou atividade produtiva quanto quais são as mais eficientes (HORA et al., 2015). 


\section{MATERIAIS E MÉTODOS}

\subsection{Classificação da Pesquisa}

De forma a classificar a pesquisa podemos afirmar que é descritiva pois, segundo Gil (2010), têm como objetivo a descrição das características de determinado fenômeno ou população, buscando identificar possíveis relações entre as variáveis.

No que se refere à natureza da sua finalidade, o trabalho aqui proposto, é considerado como uma Pesquisa Aplicada, pois é voltado para aquisição de conhecimentos decorrentes de uma aplicação numa situação específica. De acordo com Appolinário (2009), a pesquisa aplicada tende em atender objetivos comerciais, enquanto a básica está ligada ao incremento científico.

Quanto à abordagem do problema, a pesquisa é quantitativa, pois segundo Ganga (2012), esse tipo de abordagem permite quantificar e confirmar de forma estatística as relações existentes entre as variáveis da pesquisa.

E por fim pode ser considerada um estudo de caso, pois é voltada para os programas de pósgraduação stricto sensu das Engenharias III. O papel do pesquisador, num estudo de caso, é obter informações do fenômeno segundo a visão de indivíduos, bem como observar e coletar evidências que possibilitem interpretar o ambiente em que a problemática ocorre (SILVA; MENEZES, 2005; GANGA, 2012).

\subsection{Pesquisa Metodológica}

A CAPES, enquanto organismo público que emprega recursos do erário em prol da população deve ter sua gestão avaliada de forma transparente e responsavelmente. Hora et al. (2015) já utilizaram a análise envoltória de dados para avaliar a eficiência da gestão pública, e apesar de não ter sido explicitamente na área de gestão educacional, os resultados apontaram para direção da melhoria.

No mesmo propósito deste trabalho, Lins, Almeida e Bartholo Júnior (2004) utilizaram o DEA para mensurar o desempenho na pós-graduação. Trabalho semelhante foi realizado por Mello et al. (2003), onde o DEA foi utilizado para avaliar 12 programas de pós-graduação na UFRJ.

A aplicação deste instrumento é reforçada por diversos autores que utilizam desta ferramenta, como Almeida Filho e Ramos (2005) que avaliam o desempenho de forma quantitativa dos programas de pós-graduação em Engenharia de Produção das Instituições Federais de Ensino Superior (IFES) utilizando a técnica DEA, incorporando os critérios de avaliação desenvolvidos e adotados pela CAPES.

O objetivo dessa métrica é o de verificar se unidades produtivas operam de maneira adequada quando observada a relação entre um elenco específico de recursos utilizados e de resultados obtidos em unidades consideradas similares por seus administradores, sem a necessidade de conhecer, a priori, qualquer relação de importância (pesos) entre as variáveis consideradas (BELLONI, 2000). 


\subsection{Procedimentos Técnicos}

Os dados são disponíveis no portal CAPES (Coordenação de Aperfeiçoamento de Pessoal de Nível Superior) adotando o triênio 2010-2011-2012, e tornados públicos em 2013, como base da pesquisa, uma vez que se refere ao último dado disponível por ocasião desta pesquisa. Os indicadores são os presentes nas tabelas comparativas dos programas da área de Engenharias III.

A técnica a ser utilizada é o DEA que considera múltiplos recursos e múltiplos resultados sem exigir que se conheça a priori um conjunto de taxas de substituição (pesos) entre as variáveis nem a especificação da forma funcional das relações entre os recursos e os resultados. A unidade básica de observação é a universidade e as medidas de eficiência produtiva são construídas utilizando Análise por Envoltória de Dados (BELLONI,2000).

É realizado um experimento com ambas abordagens CRS (CHARNES; COOPER; RHODES, 1978) e VRS (BANKER; CHARNES; COOPER, 1984), para escolha da abordagem mais adequada, além da filosofia da avaliação. A primeira retorna cerca de $30 \%$ das DMUs na fronteira da eficiência, e a segunda cerca de 60\% (resultados em anexo).

Como o objetivo da avaliação é o discriminamento das DMUs e identificação de suas ineficiências, a abordagem CRS é mais adequada. Ademais, os retornos constantes de variáveis são preferíveis quando há presunção de heterogeneidade das alternativas avaliadas, e a intenção é produzir os melhores resultados mediante as restrições impostas pelos recursos. Adota-se a orientação à outputs uma vez que esta avalia melhores resultados com os mesmos recursos (inputs).

De acordo com Barbosa (2007), é necessário classificar as variáveis como as de entrada (input) e as de saída (output). Para avaliação da eficiência dos programas de pós-graduação, serão consideradas como variáveis de output: o número de dissertações e teses, publicações de artigos, trabalhos completos, texto integral, capítulos de livros, coletâneas e verbetes do triênio 2010-20112012. Na outra ponta do modelo, como variáveis de input, considerou-se a quantidade de professores, o número de egressos no mestrado e no doutorado assim como a maturidade de cada programa, avaliada como tempo de funcionamento em anos.

Opta-se por estudar o grupo de Engenharias III, especificamente as instituições que apresentam programas de mestrado quanto o de doutorado, totalizando 53 instituições. No entanto, nota-se à falta de dados para uma instituição, sendo esta desconsiderada neste trabalho. Portanto, serão avaliados quanto à sua eficiência, 52 programas de pós-graduação, assumindo estas o papel de unidades tomadoras de decisão (DMU’s) na modelagem da análise envoltória de dados.

Para executar os procedimentos da análise envoltória de dados é utilizado o software SIAD (Sistema Integrado de Apoio à Decisão), disponibilizado gratuitamente em: http://www.uff.br/decisao/ (ANGULO-MEZA et al., 2005).

A técnica de análise baseada nos quartis, também foi realizada de modo a obter uma análise mais individualizada dos resultados de eficiência. A técnica em questão consiste em separar os resultados em grupos ordenados.

\subsection{Limitações Metodológicas}

A limitação encontrada tange ao aspecto de caracterização da amostra, uma vez que o software utilizado (SIAD), não aceita um número maior que 100 unidades tomadoras de decisão (DMU’s) assim como o limite de variáveis que deve ser menor ou igual a 20. 
Outrossim, aponta-se que a eficiência é medida em termos dos indicadores publitizados pela CAPES, e não necessariamente em uma visão holística, que verificaria outros aspectos não abordados nesta pesquisa.

\section{RESULTADOS E DISCUSSÃO}

No presente trabalho é utilizado o modelo DEA com retornos constantes de escala (CRS), devido a heterogeneidade dos dados, assim como em decorrência de alterações que possam ocorrer nos inputs provocariam grandes impactos nos outputs. As variáveis de input e output utilizadas estão ilustradas no Quadro 1.

Quadro 1- Variáveis de Avaliação.

\begin{tabular}{|c|c|}
\hline Variáveis de entrada & Variáveis de saída \\
Inputs & Ouputs \\
\hline Quantidade de professores & Quantidade de dissertações \\
Egressos de doutorado & Quantidade de Teses \\
Egressos de mestrado & Artigos em periódico classificado (APC) \\
Maturidade do mestrado & Trabalhos completos em anais \\
Maturidade do doutorado & Capítulos de livros \\
& Coletâneas \\
& Verbetes \\
\hline
\end{tabular}

A variável de saída "Artigos em periódicos classificado" congrega todas as publicações dos sete estratos avaliadores pelo comitê de avaliação de Área, de A1; A2; B1; B2; B3; B4 e B5, de acordo com o cálculo A1-equivalente, presente no documento de área da Engenharia III (CAPES, 2013).

$$
A P C=A 1+0,85 \times A 2+0,70 \times B 1+0,50 \times B 2+0,20 \times B 3+0,10 \times B 4+0,05 \times B 5
$$

Para avaliação crítica os resultados são separados em quartis, de modo a classificar os programas de pós-graduação em quatro categorias, sendo a pior classificada como 1, e a melhor como 4.

Quadro 2- Divisão em quartis utilizada no estudo.

\begin{tabular}{|l|c|c|c|c|c|c|}
\hline Classificação 1 & Q1 & Classificação 2 & Q2 & Classificação 3 & Q3 & Classificação 4 \\
\cline { 6 - 7 } & 0,6217 & & 0,8455 & & 1,0000 & \multicolumn{1}{c}{}
\end{tabular}

Somente a título ilustrativo, e reafirmar a escolha da abordagem, o Quadro 3, que apresenta os valores delimitadores dos quartis caso fosse adotada a abordagem DEA-BCC, com retornos variáveis de escala.

Quadro 3- Divisão em quartis dos resultados da abordagem DEA-BCC, presente no Anexo II.

\begin{tabular}{|l|c|l|c|l|c|c|}
\hline Classificação 1 & Q1 & Classificação 2 & Q2 & Classificação 3 & Q3 & Classificação 4 \\
& & 0,82756 & & & 1,00000 &
\end{tabular}

Como Q2 possuiria o mesmo valor de Q1, dada a grande concentração de DMUs na fronteira de eficiência, e como consequência, figurando no melhor quartil e na melhor classificação, com pouco efeito discriminatório desejado no estudo, esta abordagem é preterida à utilizada.

Considerando o conceito atribuído pela CAPES à cada programa de pós graduação, tem-se a seguinte distribuição ilustrada na Figura 1 


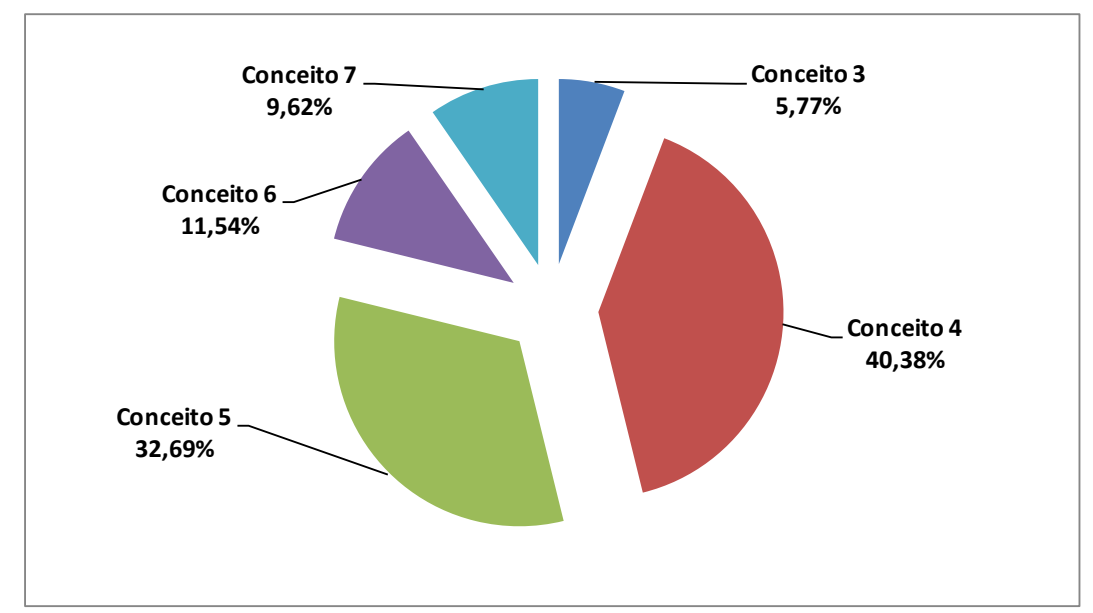

Figura 1- Distribuição dos conceitos dos programas de pós-graduação na área de Engenharia III da CAPES.

$\mathrm{Na}$ Tabela 1, pode-se notar as universidades que foram mais eficientes em relação aos seus programas de pós-graduação. São estes os programas que estão localizados na fronteira de eficiência obtendo uma eficiência Q3 $\geq 1,00$.

Tabela 1: Programas de Pós-Graduação posicionados na Classificação 4.

\begin{tabular}{ccc}
\hline PROGRAMA & EFICIÊECIA & CONCEITO CAPES \\
\hline DMU 2 & 1,00 & 4 \\
DMU 7 & 1,00 & 4 \\
DMU 10 & 1,00 & 4 \\
DMU 1 1 & 1,00 & 5 \\
DMU 12 & 1,00 & 4 \\
DMU 14 & 1,00 & 5 \\
DMU 22 & 1,00 & 6 \\
DMU 24 & 1,00 & 5 \\
DMU 27 & 1,00 & 6 \\
DMU 28 & 1,00 & 4 \\
DMU 30 & 1,00 & 5 \\
DMU 36 & 1,00 & 4 \\
DMU 38 & 1,00 & 5 \\
DMU 41 & 1,00 & 5 \\
DMU 45 & 1,00 & 5 \\
DMU 46 & 1,00 & 4 \\
DMU 51 & 1,00 & 4 \\
\hline
\end{tabular}

Destacam-se 17 programas de pós-graduação com eficiência máxima igual 1,00, dentre eles observa-se os programas de engenharia mecânica e produção de instituições de administração variada, entre estadual, federal e privada, como UERJ, PUC/RJ, UFF e USP.

Interessante notar que no quartil mais eficiente, onde todos os programas que figuram na fronteira da eficiência, não há nenhum programa considerado de qualidade internacional, representado pelo conceito 7. Outrossim, corrobora-se que os programas de doutorado com conceito 3 apresentam problemas de produção de resultados, e não figuram neste quartil.

Aponta-se ainda a presença de oito programas com conceito 4, que seria o conceito inicial de um programa de doutorado. Apesar de figurar no conceito mínimo, estes programas apresentam excelente eficiência, sendo considerados tecnicamente referências para os demais.

Pode-se verificar de acordo com a Tabela 2, os programas correspondentes a classificação 3 com valores de eficiência entre Q3 $<1,00$ e Q2 $\geq 0,8455$. 
Tabela 2: Programas de Pós-Graduação posicionados na Classificação 3.

\begin{tabular}{ccc}
\hline PROGRAMA & EFICIÊNCIA & CONCEITO CAPES \\
\hline DMU 20 & 0,9940 & 5 \\
DMU 8 & 0,9578 & 5 \\
DMU 5 & 0,9458 & 7 \\
DMU 52 & 0,9394 & 4 \\
DMU 18 & 0,9309 & 6 \\
DMU 49 & 0,9182 & 5 \\
DMU 1 & 0,8929 & 4 \\
DMU 47 & 0,8889 & 6 \\
DMU 44 & 0,8461 & 5 \\
\hline
\end{tabular}

Nesse quartil destacam-se os programas de Engenharia Mecânica da UFPR e PUC/PR e o curso de Engenharia Mecânica e Materiais da UTFPR com eficiências respectivas: 0,9940; 0,9578 e 0,9458 .

Por ser ainda uma classificação próxima à fronteira da eficiência, nutre-se a expectativa, e portanto, assume-se a hipótese de que de programas bem avaliados ainda figurarão nesta classificação. Esta hipótese é parcialmente confirmada, pela presença de um programa avaliado com o conceito 7 (DMU 5), mas também aponta-se a presença de dois programa com conceito 4 (DMU 1 e DMU 52).

A Tabela 3 aponta os programas de pós-graduação referentes a classificação 2, correspondente a $\mathrm{Q} 2 \leq 0,8455$ e $\mathrm{Q} 1 \geq 0,6217$.

Tabela 3: Programas de Pós-Graduação posicionados na Classificação 2.

\begin{tabular}{ccc}
\hline PROGRAMA & EFICIÊNCIA & CONCEITO CAPES \\
\hline DMU 32 & 0,8448 & 3 \\
DMU 21 & 0,8409 & 4 \\
DMU 15 & 0,8202 & 4 \\
DMU 34 & 0,8056 & 4 \\
DMU 37 & 0,8037 & 5 \\
DMU 35 & 0,7480 & 4 \\
DMU 43 & 0,7422 & 3 \\
DMU 25 & 0,7026 & 7 \\
DMU 50 & 0,6866 & 6 \\
DMU 42 & 0,6598 & 5 \\
DMU 16 & 0,6566 & 5 \\
DMU 3 & 0,6288 & 5 \\
DMU 33 & 0,6284 & 7 \\
\hline
\end{tabular}

Nesta classificação observa-se os programas de Engenharia de Produção da UFSCAR, Métodos Numéricos de Engenharia da UFPR e Engenharia de Produção da UFMG.

Dois programas com o conceito máximo atribuído pela avaliação da CAPES figuram nesta classificação, bem como dois programas com problemas em sua avaliação, com conceito 3 atribuído. É a classificação com maior diversidade de conceitos. Como término de análise dos quartis, observa-se na Tabela 4, os programas referentes a classificação 1, neste caso são aqueles em que a eficiência são as piores da análise, Q1 $\leq 0,6217$. 
Tabela 4: Programas de Pós-Graduação posicionados na Classificação 1.

\begin{tabular}{lcc}
\hline PROGRAMA & EFICIÊNCIA & CONCEITO CAPES \\
\hline DMU 29 & 0,6016 & 4 \\
DMU 40 & 0,5915 & 7 \\
DMU 23 & 0,5850 & 6 \\
DMU 4 & 0,5812 & 5 \\
DMU 31 & 0,5647 & 7 \\
DMU 48 & 0,5102 & 4 \\
DMU 13 & 0,4938 & 4 \\
DMU 6 & 0,4836 & 4 \\
DMU 19 & 0,4770 & 4 \\
DMU 9 & 0,4522 & 3 \\
DMU 39 & 0,4033 & 4 \\
DMU 17 & 0,3286 & 4 \\
DMU 26 & 0,3072 & 5 \\
\hline
\end{tabular}

Nesta classificação encontram-se 13 programas de pós-graduação com eficiência consideravelmente baixa. Em destaque o programa Engenharia Oceânica da UFRJ com a menor eficiência $(0,3072)$ dentre os demais. Os cursos de Engenharia Mecânica da UFPB-JP e UNICAMP estão entre os menos eficazes com eficiências: 0,3286 e 0,4033, respectivamente. Pode-se ainda observar que o programa de Engenharia de Reservatório e de Exploração da UENF também não é eficaz, uma vez que sua eficiência foi 0,4522 .

Aponta-se a presença de dois programas com conceito 7 figurando no pior quartil, além de um programa com conceito 6. Por ser a pior classificação possível, que reúne as DMUs mais distantes da fronteira da eficiência, haveria a expectativa de conter os programas com pior avaliação, especialmente os avaliados com o conceito 3, contudo, somente um destes casos figura nesta classificação.

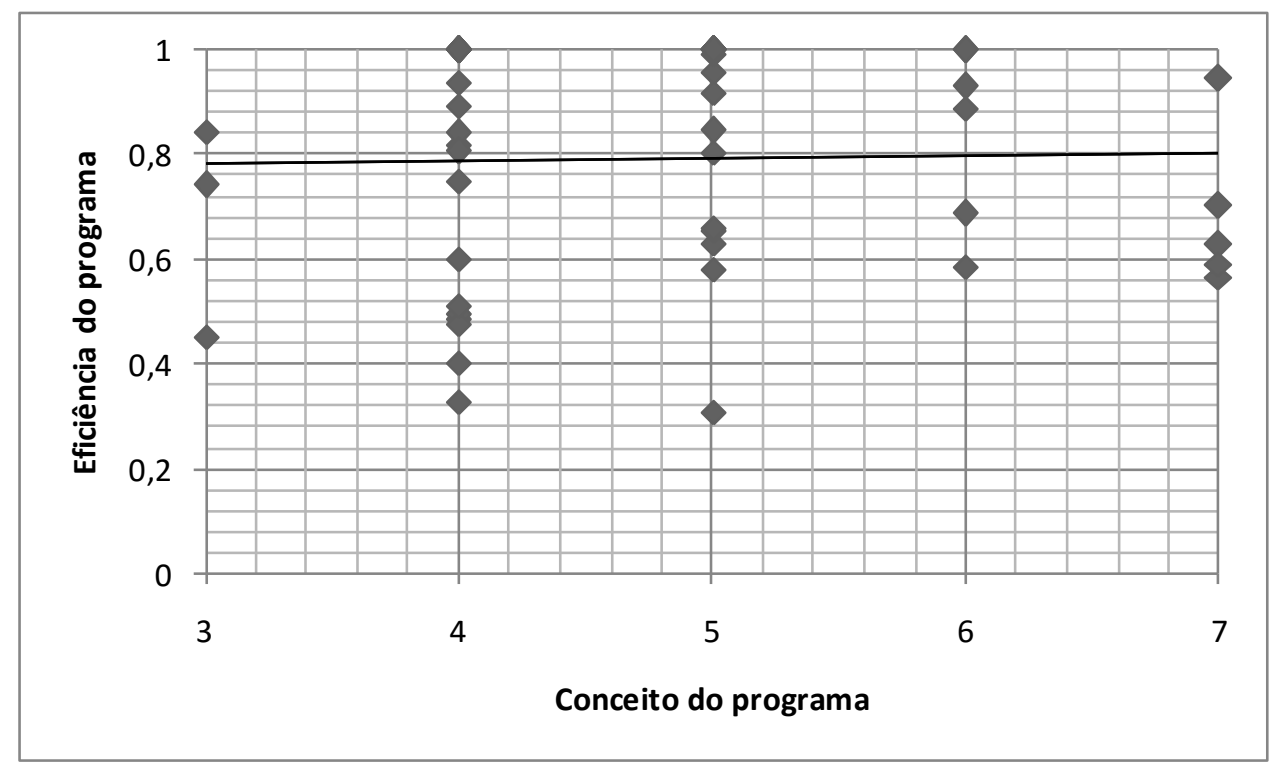

Figura 2: Gráfico de correlação entre a eficiência e o conceito do programa

A Figura 2 apresenta um gráfico de correlação entre as variáveis Conceito do programa, atribuído pela avaliação trienal 2010-2011-2012 pelo comitê avaliador das Engenharias III e a eficiência calculada pela análise envoltória de dados, modelo CCR orientado a output. $\mathrm{O}$ valor da correlação (pela forma de Pearson) é de 0,022885 , ou seja próxima de nula. 


\section{CONCLUSÃO}

Este trabalho se propôs em avaliar os indicadores de produção científica dos programas de pós graduação da área de Engenharia III da CAPES, presentes na tabela comparativa entre programas da avaliação trienal 2010-2011-2012.

Este trabalho não possui a ambição de criticar a avaliação realizada pelo comitê avaliador do triênio 2010-2011-2012, nem de propor uma avaliação paralela, mas apenas realizar um estudo de eficiência quanto aos indicadores apresentados na tabela comparativa pela própria CAPES, de modo a fornecer subsídios aos coordenadores e docentes permanentes dos programas de pós-graduação, de modo a estes concentrarem seus esforços nas ações mais valorizadas pelo comitê de avaliação.

Verifica-se que a baixa correlação entre as eficiências alcançadas e o conceito do programa fragilizam a assertiva que um programa bem avaliado é um programa com alta produção científica. Em absoluto isto não significa que a produção científica possui papel secundário na avaliação, mas que existem outros aspectos que não foram apresentados na tabela comparativa, sejam quantitativos, sejam qualitativos, que são relevantes para a atribuição do conceito final.

A análise realizada através da metodologia DEA demonstra que 17 programas de pósgraduação obtiveram a eficiência máxima, sendo altamente eficazes. A maior parte desses programas é de instituições públicas. A pesquisa ainda aponta que os programas com pior eficiência também correspondem a instituições públicas renomadas no país como UFRJ, UNICAMP, UFPBJP e UENF, o que leva uma reflexão da educação superior pública, que é heterogênea.

A presença de programas com conceito 7 em classificações consideradas ruins é um resultado que chama à atenção, uma vez que seu status de qualidade internacional gera uma expectativa de excelência em todos os indicadores, o que a pesquisa revela não ser verdade. Do mesmo modo, programas que apesar de ofertarem o doutoramento com a nota 3 , o que não é usual e demonstra que o programa possui problemas em seus indicadores, figuram nas classificações melhores.

A produção científica de um programa é transversal à avaliação, presente em diversos critérios, tanto nos para avaliação do corpo docente, do corpo discente e na produção intelectual. Esta última responde sozinha por $35 \%$ da avaliação, que somada aos indicadores das demais, ultrapassa 50\% (CAPES, 2013).

No último ano do novo período de avaliação, não mais triênio, mas agora quadriênio 20132013-2015-2016, é importante aos interessados na avaliação conhecer o máximo de faces de avaliações e interpretações da última avaliação, tanto para replicá-la na próxima avaliação, bem como para conhecer melhor os mecanismos internos de avaliação para alinhá-la em prol da ciência nacional.

Por fim, esta análise ocupou-se de avaliar o triênio 2010-2011-2012, sendo possível realizar a mesma análise para os triênios anteriores e até mesmo para outros grupos das Engenharias ou ainda verificar não somente o retrato das eficiências no ano base da pesquisa, mas também a sua evolução com o passar dos anos.

Além de replicar o método adotado neste trabalho, com as mesmas variáveis, em avaliações trienais diferentes, para verificar a evolução ao longo dos anos o desempenho de cada programa, é interessante incorporar ao modelo mais variáveis não consideradas neste estudo, para uma avaliação holística, mais próxima da realidade dos programas. 
Uma modelagem que considere amplamente os elementos considerados na avaliação, que vão além da tabela comparativa disponibilizada pela CAPES é um possível caminho de pesquisa futura, que forneceria outras informações para os coordenadores de cursos e interessados nos resultados.

\section{REFERENCIAS}

ALMEIDA FILHO, A. T.; RAMOS, F. S. Avaliação de desempenho de programas de pós-graduação com análise envoltória de dados. In: XXV - Encontro Nacional de Engenharia de Produção - ENEGEP 2005, Porto Alegre, RS. Disponível em http://www.abepro.org.br - Acesso em 13 de novembro de 2014.

ÂNGULO MEZA, L.; GOMES, E.G.; BIONDI NETO, L. Coelho, P. H. G. Avaliação do Ensino nos cursos de pósgraduação em engenharia: um enfoque quantitativo de avaliação em conjunto. Engevista, Niterói, volume 5, número 9, p.41-49. 2003.

APPOLINÁRIO, F. Metodologia da ciência: filosofia e prática da pesquisa. São Paulo: Cengage Learning, 2009.

BANKER, R. D.; CHARNES, A.; COOPER, W. W. Some Models for Estimating Technical and Scale Inefficiencies in Data Envelopment Analysis. Management Science, v. 30, n. 9, p. 1078-1092, set. 1984.

BARBOSA,S. de L. et al; Avaliação da eficiência Docente em um Programa de Pós Graduação Stricto Sensu na Região Sul do Brasil com o uso de Data Envelopment Analysis - DEA. In: XXXI Encontro da ANPAD, Rio de Janeiro, RJ. 2007.

BELLONI, J. A. Uma metodologia de avaliação da eficiência produtiva de Universidades Federais Brasileiras. Tese de Doutorado, Programa de Pós-Graduação em Engenharia de Produção da Universidade Federal de Santa Catarina (UFSC), Florianópolis, SC. Abril de 2000.

BRASIL. Decreto n 86.791 de 28 de dezembro de 1981. Plano Nacional de Pós-Graduação Stricto Sensu. Diário Oficial da União - Seção 1 - 29/12/1981, Página 24960 (Publicação Original). Disponível em: http://www2.camara.leg.br/legin/fed/decret/1980-1987/decreto-86791-28-dezembro-1981-436402-norma-pe.html. Acesso em 15 de dezembro de 2015.

BRASIL. Lei no 9.394 de 20 de dezembro de 1996. Estabelece as diretrizes e bases da educação nacional. Disponível em: http://www.planalto.gov.br/ccivil_03/leis/19394.htm - Acesso em 15 dezembro de 2015.

CAPES - Coordenação de Aperfeiçoamento de Pessoal de Nível Superior - Documento de área e Comissão da Trienal 2013. Disponível em <http://www.capes.gov.br/component/content/article/44-avaliacao/4686-engenharias-iii>, 2013, Acesso em 11 de dezembro de 2015.

CHARNES, A.; COOPER, W. W.; RHODES, E. Measuring the efficiency of decision making units. European Journal of Operational Research, v. 2, n. 6, p. 429-444, nov. 1978.

FERREIRA, C. M. de C.; GOMES, A.P. Introdução à Análise Envoltória de Dados. Teoria, modelos e aplicações. 1.ed. 1. Reimpressão. Viçosa, MG: Editora UFV. 2012.

GANGA, G. M. D. Trabalho de conclusão de curso (TCC) na Engenharia de Produção: Um guia prático de conteúdo e forma. 1. Ed. São Paulo: Atlas, 2012.

GIL, A. C. Como elaborar projetos de pesquisa. 5. ed. São Paulo: Atlas. 2010.

HORA, A. L. B. et al. Análise da eficiência dos serviços de saneamento básico nos municípios do estado do Rio de Janeiro. Pesquisa Operacional para o Desenvolvimento, v. 7, n. 1, p. 55-81, jan. 2015.

JOUMADY, O., RIS, C. Performance in European higher education: A non-parametric production frontier approach. Education Economics, 13:2, p. 189 - 205; 2005. Disponível em: www.tandfonline.com/loi/cede20. Acesso em 02 de outubro de 2015.

LINS, Marcos Pereira Estellita; ALMEIDA, Bernardo Faria; BARTHOLO JÚNIOR, Roberto. Avaliação de desempenho na pós-graduação utilizando a análise envoltória de dados: O caso da Engenharia de Produção. RBPG Revista Brasileira de Pós-Graduação, vol.1, n.1, p.41-56, jul.2004. Disponível em: http://ojs.rbpg.capes.gov.br/index.php/rbpg/article/view21 . Acesso em: 12 de novembro de 2014.

MELLO, J. C. C. B. S. DE et al. Uma análise da qualidade e da produtividade de programas de pós-graduação em engenharia. Relatórios de Pesquisa em Engenharia de Produção, v. 2, n. 1, 2003. 
MELlo, J. C. C. B. S.; ÂNGELO MEZA, L.; GOMES, E. G.; BIONDI NETO, L. Curso de Análise Envoltória de Dados. In: XXXVII SIMPÓSIO BRASILEIRO DE PESQUISA OPERACIONAL - SBPO, 2005 , Gramado - RS. Disponível em http://www.sbpo.com.br - Acesso em 15 de dezembro de 2015.

MELLO, M. H. C. S.; MELLO, J. C. C. B. S. e ÂNGELO MEZA, L. Medida de seletividade dos cursos de Engenharia da UFF com modelo DEA. Engevista, vol. 13 n 03, p. 219-255, Rio de Janeiro 2011.

MIRANDA, C. M. G.; ALMEIDA, A. T. Avaliação de pós-graduação com método ELECTRE TRI - o caso de Engenharias III da CAPES. Production, vol. 13 n 03, São Paulo 2003. Disponível em http://dx.doi.org/10.1590/S01365132003000300009 - Acesso em 13 de novembro de 2014.

MOREIRA, N. P. et al.; Fatores determinantes da eficiência dos programas de pós-graduação acadêmicos em administração, contabilidade e turismo. Avaliação, Campinas; Sorocaba, SP, v. 16, n. 1, p. 201-230, mar. 2011.

SENRA, L. F. A.; NANCI, L. C.; MELlO, J. C. C. B. S; ÂNGULO-MEZA, L. Estudo sobre Método de seleção de variáveis em DEA. Pesquisa Operacional, volume 27, número 2, p.191-207, maio a agosto de 2007

SILVA, E. L. S.; MENEZES, E. M. M. Metodologia da Pesquisa e Elaboração de Dissertação. 4. ed. Florianópolis: UFSC, 2005.

STALLIVIERI, Luciane. O Sistema de Ensino Superior do Brasil: características, tendências e perspectivas. Caxias do Sul/RS, 2009. Disponível em: http://www.ucs.br - Acesso em: 13 nov. 2014.

TURRIONI, J. B.; MELLO, C. H. P. Metodologia de pesquisa em engenharia de produção. Itajubá: UNIFEI, 2012.

VEENSTRA, C.; HERRIN,G. D. An analysis of graduation rates at research universities. American Society for Engineering Education, p. 11.172.1 - 11.172.16, 2006. Acesso em 02 de outubro de 2015. 


\section{ANEXO 1 - Lista de DMU's}

\begin{tabular}{|c|c|}
\hline 1. & Engenharia e Tecnologia Espaciais - INPE \\
\hline 2. & Ciências e Tecnologias Espaciais - ITA \\
\hline 3. & Engenharia Aeronáutica e Mecânica - ITA \\
\hline 4. & Engenharia de Produção - PUC/RJ \\
\hline 5. & Engenharia Mecânica - PUC/RJ \\
\hline 6. & Engenharia Mecânica - PUC/MG \\
\hline 7. & Engenharia de Produção e Sistemas - PUC/PR \\
\hline 8. & Engenharia Mecânica - PUC/PR \\
\hline 9. & Engenharia de Reservatório e de Exploração - UENF \\
\hline 10. & Engenharia Mecânica - UERJ \\
\hline 11. & Engenharia Industrial - UFBA \\
\hline 12. & Mecatrônica - UFBA \\
\hline 13. & Engenharia de Produção - UFF \\
\hline 14. & Engenharia Mecânica - UFF \\
\hline 15. & Engenharia de Produção - UFMG \\
\hline 16. & Engenharia Mecânica - UFMG \\
\hline 17. & Engenharia Mecânica - UFPB/J.P. \\
\hline 18. & Engenharia de Produção - UFPE \\
\hline 19. & Engenharia Mecânica - UFPE \\
\hline 20. & Engenharia Mecânica - UFPR \\
\hline 21. & Métodos Numéricos de Engenharia - UFPR \\
\hline 22. & Engenharia de Produção - UFRGS \\
\hline 23. & Engenharia Mecânica - UFRGS \\
\hline 24. & Engenharia de Produção - UFRJ \\
\hline 25. & Engenharia Mecânica - UFRJ \\
\hline 26. & Engenharia Oceânica - UFRJ \\
\hline
\end{tabular}

\begin{tabular}{|l|l|}
\hline 27. & Planejamento Energético - UFRJ \\
\hline 28. & Ciência e Engenharia de Petróleo - UFRN \\
\hline 29. & Engenharia Mecânica - UFRN \\
\hline 30. & Engenharia de Produção - UFSC \\
\hline 31. & Engenharia Mecânica - UFSC \\
\hline 32. & Engenharia de Produção - UFSCAR \\
\hline 33. & Engenharia Mecânica - UFU \\
\hline 34. & Ciências Mecânicas - UNB \\
\hline 35. & Sistemas Mecatrônicos - UNB \\
\hline 36. & Engenharia Mecânica - UNESP/BAU \\
\hline 37. & Engenharia Mecânica - UNESP/GUAR \\
\hline 38. & Engenharia Mecânica - UNESP/IS \\
\hline 39. & Ciências e Engenharia de Petróleo - UNICAMP \\
\hline 40. & Engenharia Mecânica - UNICAMP \\
\hline 41. & Engenharia de Produção - UNIFEI \\
\hline 42. & Engenharia Mecânica - UNIFEI \\
\hline 43. & Engenharia de Produção - UNIMEP \\
\hline 44. & Engenharia de Produção - UNIP \\
\hline 45. & Engenharia e Produção de Sistemas - UNISINOS \\
\hline 46. & Engenharia (Engenharia de Produção) - USP \\
\hline 47. & Engenharia Mecânica - USP \\
\hline 48. & Engenharia Naval e Oceânica - USP \\
\hline 49. & Engenharia de Produção - USP/SC \\
\hline 50. & Engenharia Mecânica - USP/SC \\
\hline 51. & Engenharia de Produção - UTFPR \\
\hline 52. & Engenharia de Mecânica e de Materiais - UTFPR \\
\hline & \\
\hline
\end{tabular}


Anexo 2 - Resultados das abordagens BCC e CCR

\begin{tabular}{|c|c|c|}
\hline DMU & BCC & CCR \\
\hline DMU 01 & 1,0000 & 0,8929 \\
\hline DMU 02 & 1,0000 & 1,0000 \\
\hline DMU 03 & 1,0000 & 0,6288 \\
\hline DMU 04 & 0,9193 & 0,5812 \\
\hline DMU 05 & 0,955531 & 0,9458 \\
\hline DMU 06 & 0,935655 & 0,4836 \\
\hline DMU 07 & 1,0000 & 1,0000 \\
\hline DMU 08 & 1,0000 & 0,9578 \\
\hline DMU 09 & 0,810241 & 0,4522 \\
\hline DMU 10 & 1,0000 & 1,0000 \\
\hline DMU 11 & 1,0000 & 1,0000 \\
\hline DMU 12 & 1,0000 & 1,0000 \\
\hline DMU 13 & 0,554605 & 0,4938 \\
\hline DMU 14 & 1,0000 & 1,0000 \\
\hline DMU 15 & 0,833333 & 0,8202 \\
\hline DMU 16 & 0,656904 & 0,6566 \\
\hline DMU 17 & 0,512011 & 0,3286 \\
\hline DMU 18 & 1,0000 & 0,9309 \\
\hline DMU 19 & 0,620697 & 0,477 \\
\hline DMU 20 & 1,0000 & 0,994 \\
\hline DMU 21 & 0,841134 & 0,8409 \\
\hline DMU 22 & 1,0000 & 1,0000 \\
\hline DMU 23 & 0,585016 & 0,585 \\
\hline DMU 24 & 1,0000 & 1,0000 \\
\hline DMU 25 & 0,716318 & 0,7026 \\
\hline DMU 26 & 0,647059 & 0,3072 \\
\hline
\end{tabular}

\begin{tabular}{|c|c|c|}
\hline DMU & BCC & CCR \\
\hline DMU 27 & 1,0000 & 1,0000 \\
\hline DMU 28 & 1,0000 & 1,0000 \\
\hline DMU 29 & 0,605715 & 0,6016 \\
\hline DMU 30 & 1,0000 & 1,0000 \\
\hline DMU 31 & 0,753712 & 0,5647 \\
\hline DMU 32 & 1,0000 & 0,8448 \\
\hline DMU 33 & 0,675676 & 0,6284 \\
\hline DMU 34 & 0,864873 & 0,8056 \\
\hline DMU 35 & 1,0000 & 0,748 \\
\hline DMU 36 & 1,0000 & 1,0000 \\
\hline DMU 37 & 1,0000 & 0,8037 \\
\hline DMU 38 & 1,0000 & 1,0000 \\
\hline DMU 39 & 0,750704 & 0,4033 \\
\hline DMU 40 & 1,0000 & 0,5915 \\
\hline DMU 41 & 1,0000 & 1,0000 \\
\hline DMU 42 & 0,804344 & 0,6598 \\
\hline DMU 43 & 1,0000 & 0,7422 \\
\hline DMU 44 & 1,0000 & 0,8461 \\
\hline DMU 45 & 1,0000 & 1,0000 \\
\hline DMU 46 & 1,0000 & 1,0000 \\
\hline DMU 47 & 1,0000 & 0,8889 \\
\hline DMU 48 & 0,94218 & 0,5102 \\
\hline DMU 49 & 0,951188 & 0,9182 \\
\hline DMU 50 & 0,943473 & 0,6866 \\
\hline DMU 51 & 1,0000 & 1,0000 \\
\hline DMU 52 & 1,0000 & 0,9394 \\
\hline
\end{tabular}

